\title{
Prevalence of aspergillus skin hypersensitivity in allergic rhinitis
}

\author{
Monika Bansal $^{1}$, Komaldeep Kaur ${ }^{1}$, Aditi Gupta ${ }^{1}$, Deepak Goyal ${ }^{2}$, Vishal Chopra ${ }^{1}$, Sanjeev Bhagat ${ }^{1}$, \\ Akhil D. Goel ${ }^{3}$ \\ ${ }^{1}$ Department of Pulmonary Medicine, Government Medical College, Patiala; ${ }^{2}$ Department of Pulmonary Medicine, TB \\ Hospital, Government Medical College, Patiala; ${ }^{3}$ Department of Community Medicine and Family Medicine, All India \\ Institute of Medical Sciences, Jodhpur, India
}

\begin{abstract}
Allergic rhinitis (AR) is a chronic allergen specific, IgE-mediated hypersensitivity disorder which significantly impairs the quality of life in affected patients. Many aeroallergens and molds are responsible for AR. This study was conducted to find prevalence of Aspergillus fumigatus skin hypersensitivity in patients of AR by skin prick test (SPT). 150 clinically diagnosed AR patients visiting our OPD were enrolled. Skin hypersensitivity for A. fumigatus was done by SPT in all the enrolled patients. Chi square test and
\end{abstract}

Correspondence: Dr Deepak Goyal, TB Hospital, Sheran Wala Gate, Patiala 147001, Punjab, India.

Mobile. +91.9417250755 .

E-mail: dggoyald@gmail.com

Key words: Allergic rhinitis; Aspergillus fumigatus; skin prick test.

Contributions: $\mathrm{MB}$, conception of study, analysis of data, drafting the work; KK, AG, DG, NG, analysis of data, revising work critically for important intellectual content; $\mathrm{VC}$, conception and design of the work, data analysis and interpretation, revising work critically for important intellectual content; SB, conception and design of the work, revising work critically for important intellectual content; ADG, analysis of data, interpretation of data for the work, revising work critically for important intellectual content. Final version of the paper was approved by all the authors. All authors agreed for the accountability for all aspects of the work regarding accuracy and integrity of study work.

Conflict of interest: The authors declare that they have no known competing financial interests or personal relationships that could have appeared to influence the work reported in this paper.

Ethics approval and informed consent: Approval for the study was taken from the Research and Institutional Ethical Committee, Government Medical College, Patiala, Punjab and informed consent for study was taken from each patient.

Received for publication: 28 May 2021.

Accepted for publication: 4 March 2021.

${ }^{\circ}$ Copyright: the Author(s), 2021

Licensee PAGEPress, Italy

Monaldi Archives for Chest Disease 2021; $91: 1416$

doi: 10.4081/monaldi.2021.1416

This article is distributed under the terms of the Creative Commons Attribution Noncommercial License (by-nc 4.0) which permits any noncommercial use, distribution, and reproduction in any medium, provided the original author(s) and source are credited.
Student's $t$-test were applied for statistical analysis. Out of 150 patients, 60 (40\%) were positive for Aspergillus fumigatus SPT. Majority of the positive patients had persistent AR and among those $39 / 60(65 \%)$ had moderate to severe persistent AR and 12/60 (20\%) had mild persistent AR. Our study concluded that there is high (40\%) prevalence of $A$. fumigatus skin hypersensitivity among AR patients. The hypersensitivity to A. fumigatus was found more in severe AR patients and it was statistically significant.

\section{Introduction}

Allergic rhinitis (AR), is an inflammatory condition of the nasal mucosa, which is associated with an IgE-mediated immune response against allergens. It is characterized by nasal symptoms including anterior or posterior rhinorrhea, sneezing, nasal blockage and/or itching of the nose [1]. It is one of the most common chronic disorders, with reported prevalence ranging from $2 \%$ to $25 \%$ in children and $1 \%$ to $>40 \%$ in adults, globally, whereas in India, prevalence of AR ranges from 20 to $26 \%$ [2,3]. Symptoms occur during two or more consecutive days for more than 1 hour on most days. Based on duration, symptoms can be intermittent ( $<4$ days/week or $<4$ weeks/year) or persistent ( $>4$ days/week or $>4$ weeks/year). Based on the absence or presence of sleep disturbance and impairment in daily activities, it can be graded as mild AR or moderate to severe AR, respectively [1].

Various aeroallergens including molds are responsible for causing and triggering AR [1,3]. Molds are ubiquitous and responsible for causing a broad spectrum of type I-IV hypersensitivity reactions [4]. Skin prick test (SPT) is the standard test used to diagnose IgE-mediated allergy [1,3]. Indications for SPT include AR, asthma, atopic dermatitis, suspected food allergies, latex allergy, and conditions in which specific $\operatorname{IgE}$ is hypothesised to play a role. Using SPT, this study was performed with the aim to determine the prevalence of Aspergillus fumigatus skin hypersensitivity in diagnosed AR patients.

\section{Materials and Methods}

It was a cross-sectional study, conducted after taking approval from ethical committee. 150 patients of clinically diagnosed AR were enrolled by simple random sampling from the department of Pulmonary Medicine, G.M.C. Patiala. Patients of all age groups with 2 or more clinical features of AR (nasal congestion, rhinorrhea, postnasal drip, sneezing, and/or nasal itchiness) were includ- 
ed. Patients were classified clinically as per the "Allergic Rhinitis and its Impact on Asthma" (ARIA) 2008 update. Patients taking drugs that may interfere with SPT within one week of test were not enrolled. These drugs include antihistamines, H2 receptor blockers, tricyclic antidepressants, steroids, beta blockers.

SPT was performed in these patients to look for Aspergillus fumigatus hypersensitivity. A single droplet of solution containing A. fumigatus reagent was pricked on the skin of volar aspect of forearm. Histamine dichloride $(10 \mathrm{mg} / \mathrm{ml})$ and buffered glycerinated saline were used as positive and negative controls, respectively. Evaluation of skin reactivity was done by calculating the mean diameter as $(\mathrm{D}+\mathrm{d}) / 2$; where $\mathrm{D}=$ longest diameter and $\mathrm{d}=$ perpendicular diameter at the largest width of D. Redness and swelling i.e., wheal with flare, equal to or more than $3 \mathrm{~mm}$ than negative control was considered positive after $20 \mathrm{~min}$.

Statistical analysis was conducted with statistical package for social science system (SPSS version 23). Chi-square test and Student's $t$-test were applied and p-value less than 0.05 was considered significant.

\section{Results}

Majority of the patients were in 21-to-30-year age group and the mean age of presentation was 26.8 years (age ranging from 11 to 70 years). Male to female ratio was 0.82 , with female preponderance. Family history of AR was present in only 24 (16\%) patients and nearly one third (42/150) had history of asthma. Moderate to severe persistent AR (65.3\%) was the most common type of AR observed. 60 patients out of 150 patients (40\%) had positive SPT and 20 out of them had history of bronchial asthma (BA). Out of these SPT positive patients, $39(65 \%)$ had moderate to severe persistent AR, 12 (20\%) had mild persistent AR and $9(15 \%)$ had moderate to severe intermittent AR. None of the patients with mild intermittent AR were SPT positive for A. fumigatus. Hence, majority of SPT positive patients had persistent AR and among them majority had moderate to severe persistent AR. Relationship between severity of AR and presence of skin hypersensitivity to A. fumigatus was statistically significant $(\mathrm{p}<0.05)$ (Table 1$)$.

\section{Discussion}

Allergic rhinitis is an IgE-mediated hypersensitivity disorder affecting the nasal lining and characterized by nasal congestion, rhinorrhea, sneezing, nasal itchiness, and/or post nasal drip. Different type of aeroallergens including molds have dominant role in etiopathogenesis of AR.

Fungus Aspergillus comprises of 132 different species which are distributed ubiquitously in the environment and it releases large quantities of small conidiospores of $2-3 \mu \mathrm{m}$. It represents a dominant indoor as well as outdoor pathogen. Because of its thermo-tolerance, it can grow at human body temperature, and has resistance to oxidative killing and produces small metabolites and enzymes with proteolytic and immunosuppressive activity. Human disorders caused by Aspergillus range from colonization of the respiratory tract, hypersensitivity pneumonitis, allergic rhinitis, sinusitis, allergic bronchopulmonary aspergillosis and asthma, to life-threatening systemic invasive aspergillosis, about $80 \%$ of Aspergillus related infections are caused by A. fumigatus [4].

The diagnosis of allergic rhinitis is based upon the history of allergic symptoms and supportive diagnostic tests. Diagnostic tests include serum total IgE level, allergen specific IgE level and immediate hypersensitivity skin test. Serum $\operatorname{IgE}$ assay is more specific but costly as well as results are not available immediately and in contrast skin hypersensitivity testing is cheap, quick and sensitive [5]. Total serum IgE measurement has a poor predictive value for allergy screening in rhinitis. Various skin hypersensitivity testing methods are used including scratch test, prick and puncture test, intradermal skin test, prick-prick tests and atopy patch tests [1].

In this study, majority of AR patients were having moderatesevere persistent $A R$, while few had mild intermittent AR in concordance with results of other authors.[6] The severity of AR has great impact on quality of life (QOL) and there are tools available to access health related quality of life (HRQoL) in patients of AR and/or BA e.g. rhinitis and asthma patient's perspective (RAPP), concomitant asthma and rhinitis (CARAT) and asthma control test (ACT).[7] In the present study, 60 (40\%) patients were hypersensitive to A. fumigatus by SPT (Table 1). Aggarwal et al observed SPT positivity for fungi in AR patients being 9.7\%, Sharma et al. found in $8.3 \%$ and by Chogtu et al. in $13.92 \%$ [3,8,9]. The percentage of skin prick hypersensitivity to A. fumigatus has been found to be higher in our study. This can be attributed to the fact that majority of our patients were from rural population with farming background.

Special attention is needed in management of patients with concomitant $\mathrm{AR}$ and $\mathrm{BA}$ as the coexistence may impair asthma control causing more frequent asthma exacerbations and asthmarelated hospitalizations. Prevalence of BA in AR was $28 \%$ $(42 / 150)$, whereas other authors reported prevalence ranging from $16 \%$ to $55 \%$ [3,10-13]. Out of 60 SPT positive patients, 20 had concomitant BA and AR. Prevalence of BA in AR was found more

Table 1. Distribution of Aspergillus fumigatus skin hypersensitivity by SPT in allergic rhinitis patients.

\begin{tabular}{|c|c|c|c|c|c|}
\hline Aspergillus fumigatus & & Type of & gic rhinitis & & \\
\hline $\begin{array}{l}\text { skin hypersensitivity } \\
\text { by skin prick test }\end{array}$ & Mild intermittent & Mild persistent & $\begin{array}{l}\text { Moderate to severe } \\
\text { intermittent }\end{array}$ & $\begin{array}{c}\text { Moderate to severe } \\
\text { persistent }\end{array}$ & $\mathrm{n}=\mathbf{1 5 0}$ \\
\hline Negative & $\begin{array}{c}10 \\
(11.1 \%)\end{array}$ & $\begin{array}{c}15 \\
(16.7 \%)\end{array}$ & $\begin{array}{c}6 \\
(6.7 \%)\end{array}$ & $\begin{array}{c}59 \\
(65.6 \%)\end{array}$ & $\begin{array}{c}90 \\
(60 \%)\end{array}$ \\
\hline Positive & $\begin{array}{c}0 \\
(0.0 \%)\end{array}$ & $\begin{array}{c}12 \\
(20.0 \%)\end{array}$ & $\begin{array}{c}9 \\
(15.0 \%)\end{array}$ & $\begin{array}{c}39 \\
(65.0 \%)\end{array}$ & $\begin{array}{c}60 \\
(40 \%)\end{array}$ \\
\hline Chi-square & & & 9.391 & & \\
\hline $\mathrm{p}$ & & & 0.025 & & \\
\hline Significance & & & Significant & & \\
\hline
\end{tabular}


in our study as patients were enrolled from a tertiary care chest hospital.

As the study sample size was small, thus the results cannot be generalized, this is the limitation of our study.

\section{Conclusions}

Identification of particular allergen may be important for treatment of AR by avoidance of allergen and immunotherapy. We found a high prevalence of Aspergillus fumigatus hypersensitivity among AR patients and there was a statistically significant correlation between severity of $\mathrm{AR}$ and hypersensitivity to $A$. fumigatus by SPT, thus AR patients having hypersensitivity to A. fumigatus are more likely to have moderate to severe form of allergic rhinitis. It is suggested that all moderate to severe AR patients should be screened for $A$. fumigatus hypersensitivity.

\section{References}

1. Bousquet J, Khaltaev N, Cruz AA, et al. Allergic rhinitis and its impact on asthma (ARIA) 2008 update (in collaboration with the World Health Organization, GA2LEN and AllerGen). Allergy 2008;63:8-160.

2. Brozek JL, Bousquet J, Agache I, et al. Allergic rhinitis and its impact on asthma (ARIA) guidelines-2016 revision. J Allergy Clin Immunol 2017;140:950-8.

3. Aggarwal D, Abhilash S, Kapur S, Gupta D. Study of causal aeroallergens in allergic rhinitis. Int J Otorhinolaryngol Head Neck Surg 2019;5:916-21.
4. Simon-nobbe B, Denk U, Poll V, et al. The spectrum of fungal allergy. Int Arch Allergy Immunol 2008;145:58-86.

5. Bignardi D, Comite P, Mori I, et al. Allergen-specific IgE: comparison between skin prick test and serum assay in real life. Allergol Select 2019;3:9-14.

6. Ibekwe PU, Ibekwe TS. Skin prick test analysis in allergic rhinitis patients: a preliminary study in Abuja , Nigeria. J Allergy 2016;2016:1-5.

7. Todo-Bom A, Braido F, Molinengo G, et al. The Portuguese version of rhinitis and asthma patient's perspective (RAPP): validation and assessment. Pulmonology 2020;26:73-7.

8. Sharma RK, Mathur Y, Chhabra G, et al. A study of skin sensitivity to various allergens by skin prick test in patients of bronchial asthma and allergic rhinitis. Indian J Allergy Asthma Immunol 2018;32:47-53.

9. Chogtu B, Magaji N, Magazine R, Acharya PR. Pattern of allergen sensitivity among patients with bronchial asthma and/or allergic rhinosinusitis in a tertiary care centre of southern India. J Clin Diagn Res 2017;11:OC01-4.

10. Hakkim FA, Rajesh V, Giridhar, et al. A study on profile of allergens and the severity of FEV1 in allergy patients in a tertiary care centre in Mangalore, India. Int J Med Res Health Sci 2018;7:135-40.

11. Dhanya EK, Rajesh V, Augustine J, et al. Sensitivity pattern to common inhalant allergens in subjects with allergic rhinitis in Kerala. Eur J Pharm Med Res 2019;6:584-7.

12. Sinha B, Vibha, Singla R, Chowdhury R. Allergic rhinitis: A neglected disease - a community based assessment among adults in Delhi. J Postgrad Med 2015;61:169-75.

13. Dave L, Srivastava N. A study of sensitisation pattern to various aero-allergens by skin prick test in patients of united airway disease (UAD) in Bhopal, Madhya Pradesh, India. Res J Pharm Biol Chem Sci 2014;5:1397-403. 